\title{
Therapeutic Management of Endometritis in a Buffalo: A Case Report
}

\author{
B. R. Babji* , G. Abhinav Kumar Reddy, G. Ambica and S. Ranjith Kumar
}

Department of Veterinary Medicine, PVNR TVU Rajendranagar, Hyderabad, India

*Corresponding author

\begin{tabular}{l} 
K e y w o r d s \\
Buffalo, \\
$\begin{array}{l}\text { Endometritis, White } \\
\text { side test, Diagnosis } \\
\text { and treatment }\end{array}$ \\
Article Info \\
\hline $\begin{array}{l}\text { Accepted: } \\
\text { 18 March } 2020 \\
\text { Available Online: } \\
10 \text { April } 2020\end{array}$ \\
\hline
\end{tabular}

\section{A B S T R A C T}

\begin{abstract}
This study reports the prompt actions taken to diagnose and treats clinical case of endometritis in a buffalo. A 6 year old, Murrah buffalo was presented to the Veterinary Clinical Complex (VCC), College of Veterinary Science, Rajendranagar, and Hyderabad with a primary history of inappetence. Clinical evaluation revealed pyrexia and animal was dull and depressed with pale mucous membranes. The most prominent abnormality observed was the yellowish mucopurulent discharged from the vulval opening and per rectal palpation of uterus revealed doughy in consistency. Cervical mucus was collected and subjected to White side test and cultural examination. The predominant bacteria found were Escherichia coli and Staphylococcus aureus and also subjected to antibiotic sensitivity test and was found sensitive to Amikacin. Haemato-biochemical analysis revealed monocytosis, slightly elevated AST, hyperglobulinemia and hypoalbuminemia. Based on the history, clinical examination and laboratory findings the case was diagnosed as clinical endometritis. First two days given the symptomatic treatment for pyrexia and actual treatment was given from third day onwards. The treatment was given with Inj. Flunixin meglumine $1.1 \mathrm{mg} / \mathrm{kg}$ was administered twice a day i.m for 3 days as anti-inflammatory, anti-pyretic and analgesic and given broad spectrum short acting antibiotic Inj. Amikacin sulphate @ $22 \mathrm{mg} / \mathrm{kg}$ once daily $\mathrm{i} / \mathrm{m}$ for 5 days in order to treat current infections. Inj. Ferritas@1ml/10kg was also administered i/m once as iron supplement and other supportive medication was given. Further, intrauterine wash was performed with $0.9 \% \mathrm{NaCl}$ using a modified intravenous line passed through the vulva into the uterine body and mucopurulent discharge was irrigated by continuous flushing and $20 \mathrm{ml}$ of Amikacin was flushed into the uterine body as the final lavage. Buffalo started showing improvement from next day onwards and on recovered completely by $6^{\text {th }}$ day of post treatment which was confirmed by negative result of vaginal mucous white side test.
\end{abstract}

\section{Introduction}

Buffaloes are the main source of good quality meat and milk in India and some other developing countries despite this species is mostly reared under harsh socioeconomic conditions and shows low productive and reproductive potentials (Azawi, et al., 2008). The prevalence rate of uterine infection in buffalo- cows is much higher than in cows (Azawi, 2010) and uterine infection is one of the most important reproductive disorders in buffalo-cows (Blood, et al., 2011; Galvao, 2011). It causes high economic losses (Azawi, et al., 2008) due to its high medication as well as prolonged days open and calving intervals. Uterine function is often compromise by bacterial contamination of the uterine lumen 
during and after calving whereas, pathogenic bacteria frequently persist, causing uterine disease, a key cause of infertility (Gurcharan, et al., 2003). These pathogenic bacteria cause endometritis and in addition, it was reported that uterine bacterial infection, bacterial products or the associated inflammation, have negative effect on the higher centres and perturbs ovarian function and consequently fertility in bovines (Amiridis et al., 2003 \& Kahn, et al., 2005).

Endometritis is the inflammation of the endometrium, the mucous membrane lining of the uterus (Blood, et al., 2011) and it is the result of nonspecific because vagina is a host to numerous microorganisms where the opportunistic pathogens may invade the uterus during coitus or during postpartum period where the cervix is dilated (Kahn et al., 2005).

\section{Materials and Methods}

A 6-year old, Murrah buffalo was presented to the Veterinary Clinical Complex (VCC), College of Veterinary Science, Rajendranagar, Hyderabad with a primary history of inappetence. Detailed examination revealed elevated body temperature and buffalo was dull and depressed with pale mucous membrane. The most prominent abnormality observed was the yellowish mucopurulent discharged from the vulva opening (Fig. 1).

For detailed investigation, rectal examination was performed and found doughy uterus on palpation. Mucopurulent discharged from the cervix was collected and performed White side test (Fig. 2), by collecting $1 \mathrm{ml}$ cervical mucus in a test tube and add $1 \mathrm{ml}$ of $5 \%$ Sodium Hydroxide solution in that test tube and then heat up to boiling point and cool it under tap water. Cultural examination for bacteria isolation and also antibiotic sensitivity test to know the antibiotic of choice.

Cultural examination was done at Department of Veterinary Microbiology, College of Veterinary Science, Rajendranagar, Hyderabad with MacConkey agar for Escherichia coli (Fig. 4) and Mannitol salt agar for staphylococcus aureus (Fig. 3). Gram staining was done to identify bacteria especially, Escherichia coli (Fig. 5) and staphylococcus organisms. Antibiotic sensitivity test was done with antibiotic discs of Streptomycin (S10), Amikacin (AN30), Ofloxacin (OF2), Ceftriaxone (CTR), Enrofloxacin (EX5) and Ciprofloxacin(CIP5) placed on Muller Hinton Agar (MHA) plates with definite distance and incubated for 24hours. White side test and Haemato biochemical analysis was carried out in the diagnostic laboratory, Department of Veterinary Clinical Complex College of Veterinary Science, Rajendranagar, Hyderabad.

\section{Results and Discussion}

White side test that was done with cervical mucus showed change in colour from colourless to yellow indicating positive for endometritis. The predominant bacteria found in cultural examination were Escherichia coli and Staphylococcus aureus and antibiotic sensitivity was found sensitive to Amikacin among all the discs.

Haemato-biochemical analysis revealed monocytosis, slightly elevated AST, hyperglobulinemia and hypoalbuminemia. Based on the history, clinical examination and laboratory findings, confirmatory diagnosis of the case was done as clinical endometritis. First two days animal was given symptomatic treatment for pyrexia. Actual treatment was started after the ABST result. The treatment was given with Inj. Flunixin meglumine 
$1.1 \mathrm{mg} / \mathrm{kg}$ was administered twice a day i.m for 3 days as anti-inflammatory, anti-pyretyic and analgesic and given broad spectrum short acting antibiotic Inj. Amikacin sulfate @ $22 \mathrm{mg} / \mathrm{kg}$ once daily i.m for 5 days in order to treat current infections. Inj. Ferritas @ $1 \mathrm{ml} / 10 \mathrm{~kg}$ was also administered i.m once as iron supplement and other supportive medication with Inj. Avil-15 ml I/M, Inj. Toxol- $20 \mathrm{ml} \mathrm{I} / \mathrm{M}$ for 5 days was given.

Further, intrauterine wash was performed with $0.9 \% \mathrm{NaCl}$ using a modified intravenous line passed through the vulva into the uterine body and mucopurulent discharge was irrigated by continuous flushing and $20 \mathrm{ml}$ of Amikacin was flushed into the uterine body as the final lavage (Fig. 6). Buffalo started showing improvement from next day onwards and on recovered completely by $6^{\text {th }}$ day of post treatment which was confirmed by negative result of vaginal mucous white side test (Fig. 7).

Uterine diseases can be categorized into puerperal metritis, clinical metritis, clinical endometritis and subclinical endometritis (GalvaoK.N 2011) ${ }^{4}$. Puerperal metritis is characterized by enlargement of the uterus with fetid watery red brown uterine discharge and sign of systemic illness such as fever.

Buffalo with enlarged uterus and purulent uterine discharge but without systemic signs are classified as clinical metritis. Clinical endometritis happens when the uterine discharge is mucopurulent which was observed in this case report. In contrast, subclinical endometritis does not have any uterine discharge but can be diagnosed with presence of $>18 \%$ neutrophils through uterine cytology.

Few studies had identified Escherichia coli, Arcanobacterium pyogenes, Klebsiella spp, Proteus spp, Psedomonas spp, Clostridium spp, Staphyloccocus spp and other gramnegative anaerobic organism as the causative agents for clinical endometritis andhowever, the clinical findings of pyretic, dull and depressed with pale mucous membrane in this case were not supported stating that cow affected with endometritis rarely exhibit any systemic signs of illness plus appetite and milk production are usually unimpaired (Kahn et al., 2005) ${ }^{7}$.

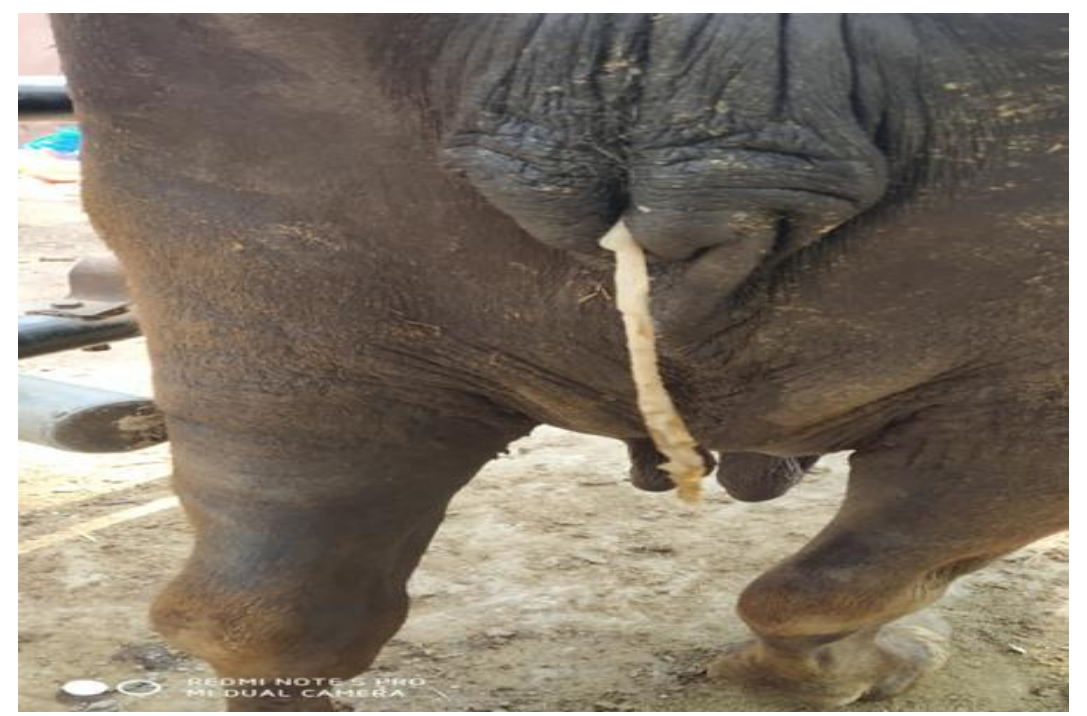

Fig.1 Yellowish mucopurulent discharge from the opening of the vulva 


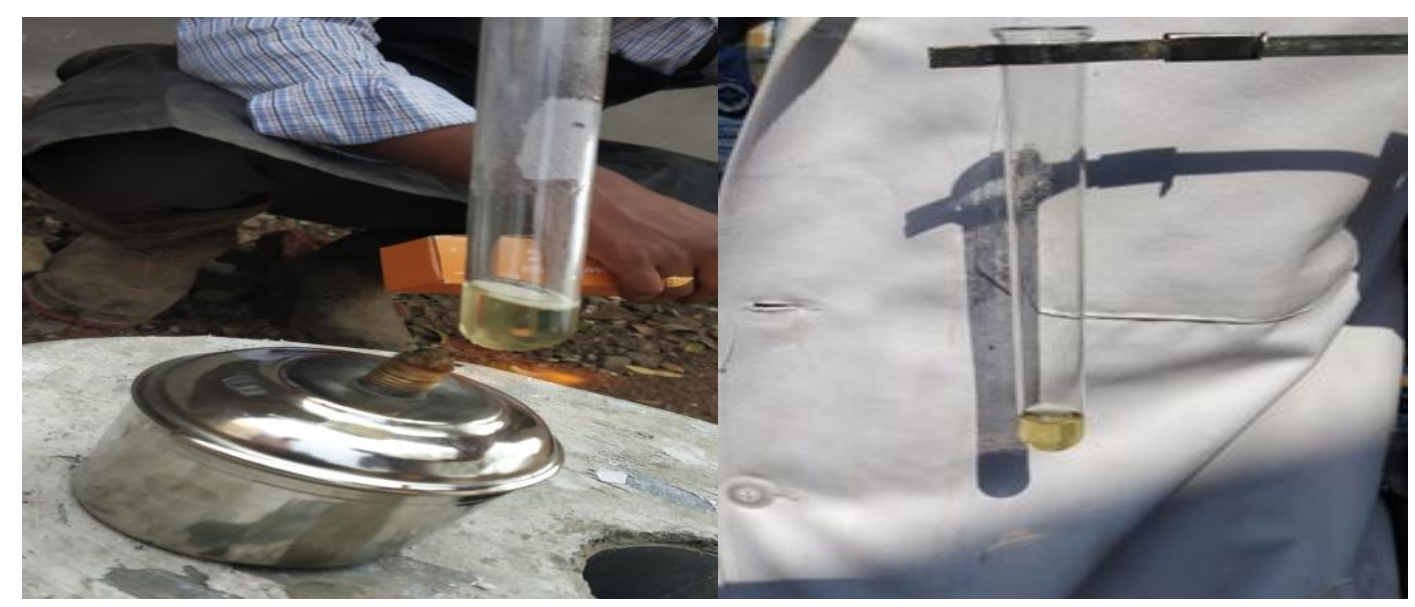

Fig.2 White side test-Changing from colourless to yellow colour indicates positive for endometritis

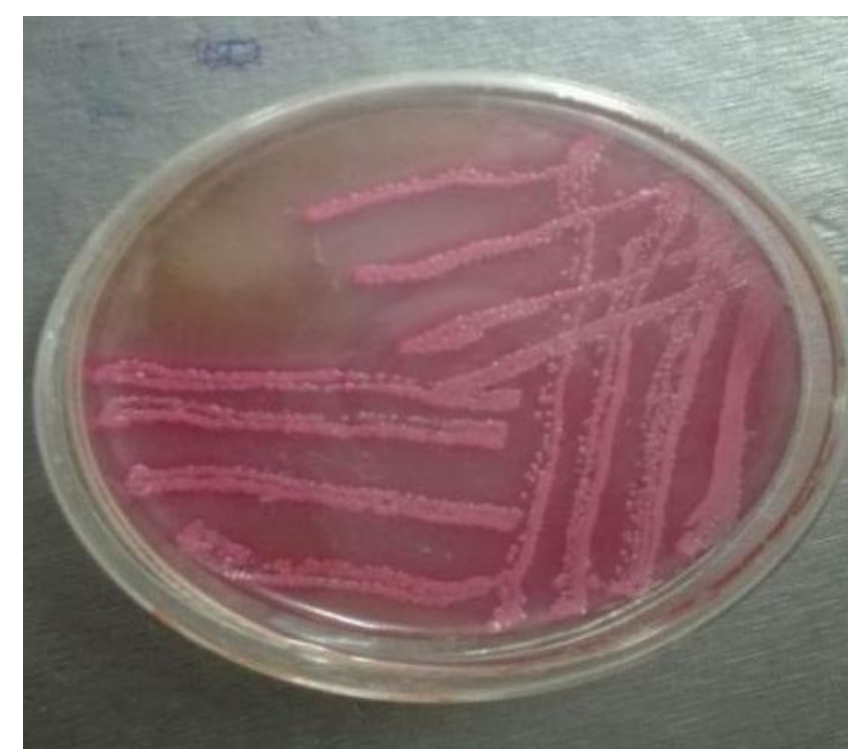

Fig.3 Escherichia coli colonies on MacConkey agar (characteristic pink colour colonies)

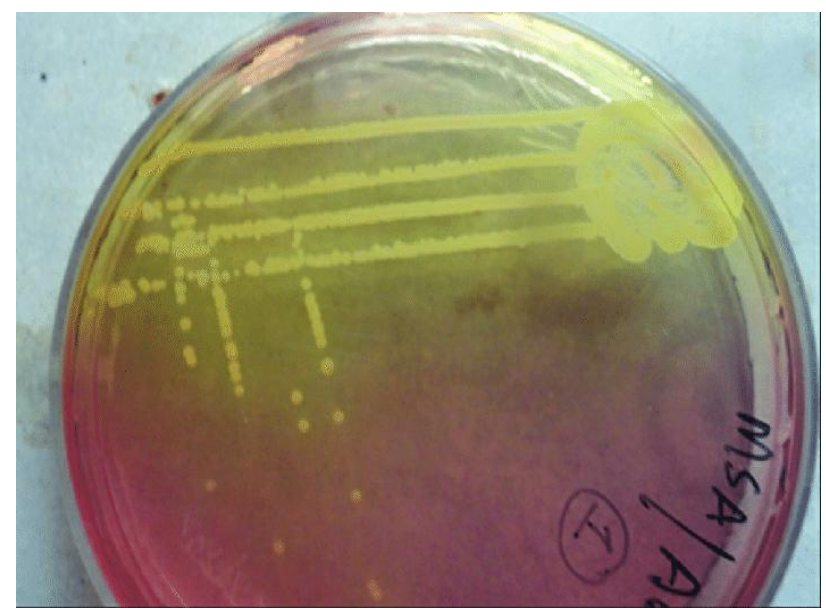

Fig.4 Staphylococcus aureus colonies on Mannitol salt agar 


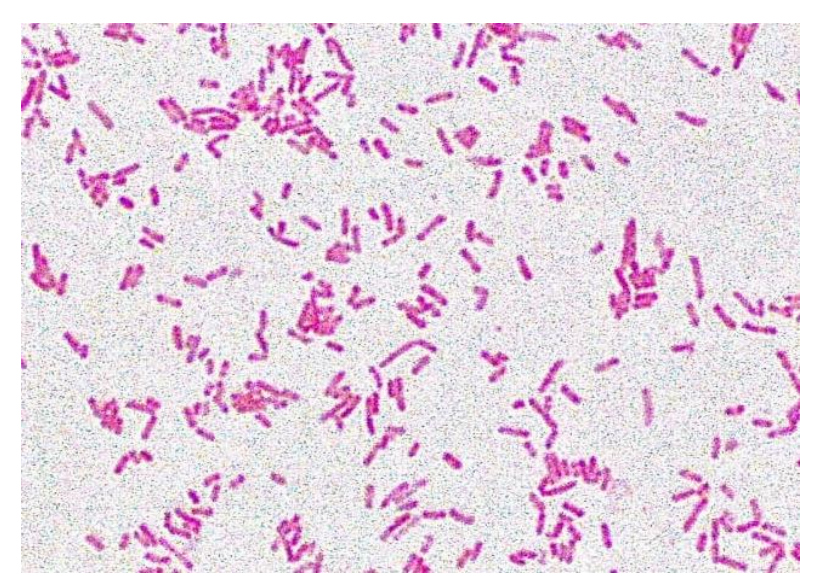

Fig.5 Escherichia coli in gram staining (pink coloured organisms)

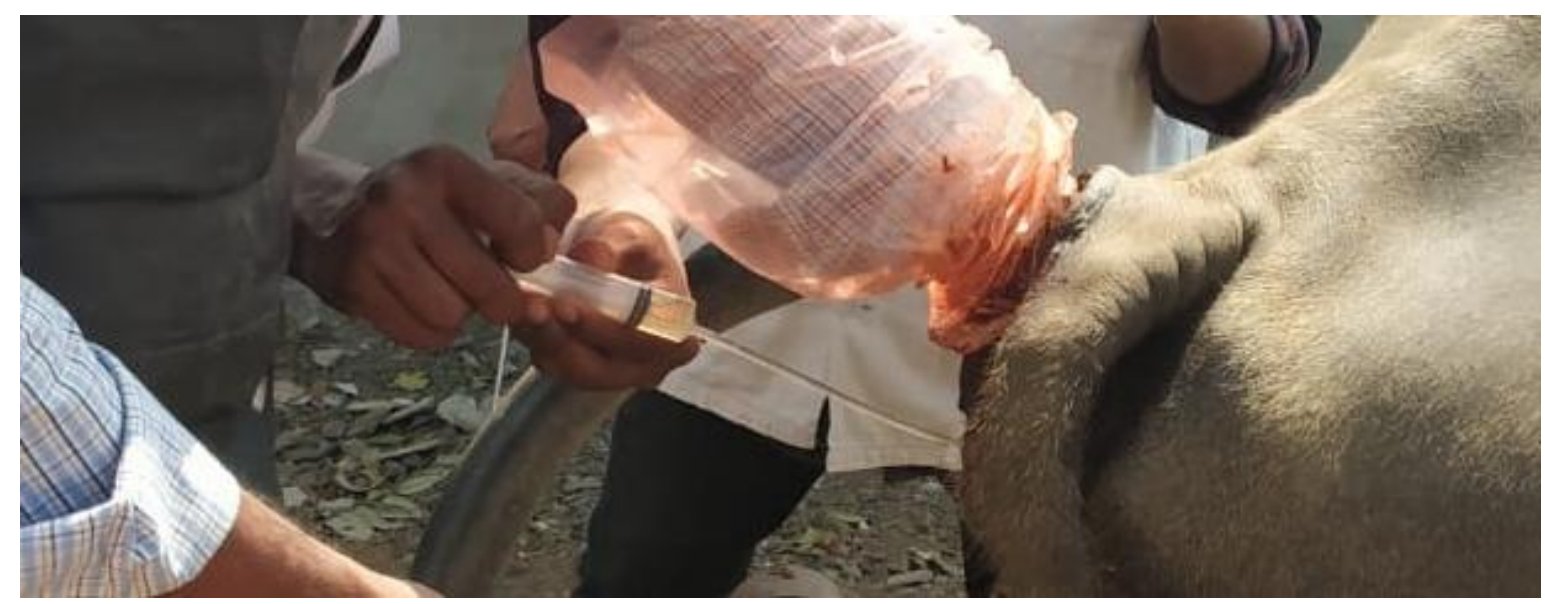

Fig.6 Intrauterine flushing of $0.9 \% \mathrm{NaCl}$ using a modified intravenous line passed through the vulva into the uterine body

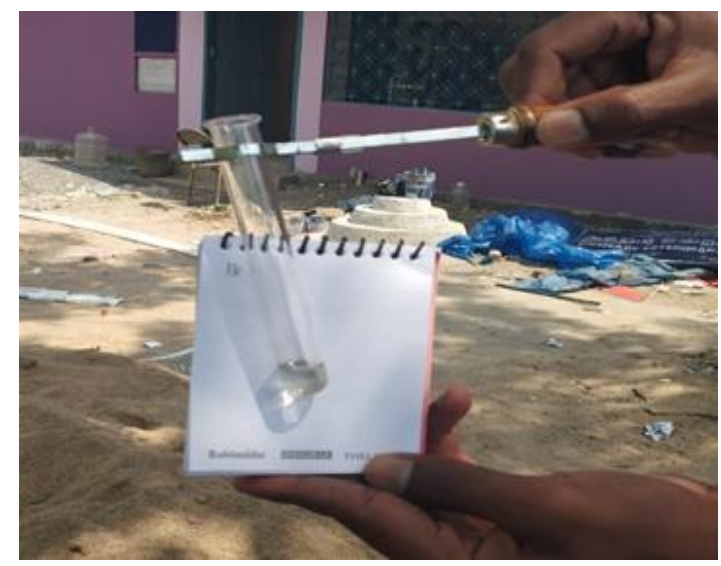

Fig.7 Post Treatment- White Side Test - No colour change, negative for endometritis

Hematology and biochemistry findings of monocytosis could be due to chronic infection and inflammation disrupting the production of red blood cells (Amiridis et al., 2003). These findings are in correlation with the previous studies sating that inflammation is able to reduce red blood count leading to anaemia (Kahn et al., 2005) ${ }^{7}$. 
With the present findings it can be concluded that Endometritis is one of the most frequent uterine disorders in postpartum dairy buffaloes causing decreased fertility leading to high economic losses and White side test can be done as a cost effective field test for the diagnosis of endometritis. Further it was evident that Intra uterine flushing of $0.9 \%$ $\mathrm{NaCl}$ and subsequent final intra uterine administration of antibiotic along with the other parenteral medication will have excellent ameliorating affect over the infection.

\section{Acknowledgement}

The authors wish to express sincere thanks to staff of Dept. of Microbiology, Veterinary Clinical Complex and students of College of Veterinary Science, Hyderabad for providing the support and technical assistance in completion of this study.

\section{References}

Azawi, O.I., S.N.Omran and J.J. Hadad. 2008. A Study of endometritis causing repeat breeding of cycling Iraqi buffalo cows. Reprod. Domest. Anim., 43: 735-743.

Azawi, O.I. 2010. Uterine infection in buffalo cows: a review. Buffalo Bull., 29: 154171.

Blood DC, Studdert VP, Gay CC (2011). Saunders comprehensive veterinary dictionary (4th Ed.,). Saunders, London.

Galvao KN (2011). Identifying and treating uterine disease in dairy cows. Proceeding 47th Florida Dairy Prod. Conf., Gainesville

Gurcharan, S., S.S. Sidhu and H.K. Verma. 2003. Incidence of reproductive disorders of buffaloes in different zones of Punjab state. Punjab Agri. Uni. J. Res., 40: 79-80.

Amiridis, G.S., G.C. Fthenakis, J. Dafopoulus, T. Papanikolaou and V. S. Mavrogianni, Use of cefquinome for prevention and treatment of bovine endometritis, Journal of Veterinary Pharmacology and Therapeutics, 26, 5, (387-390), (2003).

Kahn CM, Line S (2005). The Merck veterinary manual 9th Ed., Merial, USA.

\section{How to cite this article:}

Babji, B. R., G. Abhinav Kumar Reddy, G. Ambica and Ranjith Kumar, S. 2020. Therapeutic Management of Endometritis in a Buffalo: A Case Report. Int.J.Curr.Microbiol.App.Sci. 9(04): 2292-2297. doi: https://doi.org/10.20546/ijcmas.2020.904.274 